\title{
Analysis of Biodistribution of Intracranially Infused Radiolabeled Interleukin-13 Receptor-Targeted Immunotoxin IL-13PE by SPECT/CT in an Orthotopic Mouse Model of Human Glioma
}

\author{
Akiko Suzuki ${ }^{1}$, Pamela Leland ${ }^{1}$, Hisataka Kobayashi ${ }^{2}$, Peter L. Choyke ${ }^{2}$, Elaine M. Jagoda ${ }^{2}$, Tomio Inoue ${ }^{3}$, Bharat H. Joshi ${ }^{1}$, \\ and Raj K. Puri ${ }^{1}$ \\ ${ }^{1}$ Center for Biologics Evaluation and Research, Food and Drug Administration, Bethesda, Maryland; ${ }^{2}$ National Cancer Institute, \\ National Institutes of Health, Bethesda, Maryland; and ${ }^{3}$ Department of Radiology, Yokohama City University, Yokohama, Japan
}

\begin{abstract}
Interleukin-13 Pseudomonas exotoxin (IL-13PE), a targeted agent for interleukin-13 receptor a2 (IL-13Ra2)-expressing tumors, has been administered intracranially by convection-enhanced delivery (CED) for glioma therapy in several clinical trials including a randomized phase 3 clinical trial. However, its intracranial distribution was not optimally evaluated. We investigated the intracranial distribution of radiolabeled IL-13PE after CED in a murine model of glioblastoma multiforme. Methods: IL-13PE was radiolabeled with $\mathrm{Na}^{125}$ and evaluated for its activity in vitro in receptor-positive U251 or -negative T98G human glioma cell lines. Gliomas were grown in nude mice after intracranial implantation with U251 cells, and ${ }^{125}$ I-IL-13PE was stereotactically administered by bolus or CED for $3 \mathrm{~d}$, followed by micro-SPECT/CT imaging. SPECT images were evaluated quantitatively and compared with histology and autoradiography results. Results: The radioiodination technique resulted in a specific and biologically active ${ }^{125} \mathrm{I}-\mathrm{IL}-13 \mathrm{PE}$, which bound and was cytotoxic to IL-13Ra2-positive but not to IL-13Ra2-negative tumor cells. Both the binding and the cytotoxic activities were blocked by a 100 -fold excess of IL-13, which indicated the specificity of binding and cytotoxicity. SPECT/CT imaging revealed retention of $125 \mathrm{I}-\mathrm{IL}-13 \mathrm{PE}$ administered by CED in U251 tumors and showed significantly higher volumes of distribution and maintained detectable drug levels for a longer period of time than the bolus route. These results were confirmed by autoradiography. Conclusion: IL-13PE can be radioiodinated without the loss of specificity, binding, or cytotoxic activity. Intracranial CED administration produces a higher volume of distribution for a longer period of time than the bolus route. Thus, CED of IL-13PE is superior to bolus injection in delivering the drug to the entire tumor.
\end{abstract}

Key Words: SPECT; brain tumor; convection-enhanced delivery; biodistribution; IL-13PE; radioiodination

J Nucl Med 2014; 55:1323-1329

DOI: 10.2967/jnumed.114.138404

Received Feb. 3, 2014; revision accepted May 1, 2014

For correspondence or reprints contact: Raj K. Puri, Division of Cellular and Gene Therapy, Center for Biologics Evaluation and Research, Food and Drug Administration, National Institutes of Health Building 29B, Rm. 2NN20, 29 Lincoln Dr., Bethesda, MD 20892.

E-mail: raj.puri@fda.hhs.gov

Published online Jun. 19, 2014.

COPYRIGHT (C 2014 by the Society of Nuclear Medicine and Molecular Imaging, Inc.
$\mathbf{R}$ ecurrent glioblastoma multiforme (GBM) remains challenging to treat because of its diffuse and infiltrative nature, its cytologic heterogeneity, and the inability to adequately deliver drugs at therapeutic doses. Despite treatment with surgery, radiation therapy, and chemotherapy, prognosis remains poor, particularly for GBM, with a median survival of less than $1 \mathrm{y}$ at initial diagnosis and 6-8 mo after recurrence or progression (1). To target GBM without damaging adjacent normal tissue and achieve therapeutic concentrations of the drug, locoregional delivery methods such as convection-enhanced delivery (CED) or biodegradable polymer implantation have been developed and shown promising results in preclinical studies and early phase clinical trials (2,3). CED relies on a continuous pressure gradient to administer a therapeutic agent directly into the interstitial space of the brain over a few days to bypass the blood-brain barrier and increase drug distribution to the target tissue. Despite the appeal of delivering therapy directly to the site of disease, many phase 2 and 3 clinical trials using CED for GBM have failed to achieve endpoint objectives partly due to uncertainties of drug delivery to the entire tumor $(4,5)$.

Cintredekin besudotox, also known as IL-13PE, is a recombinant chimeric cytotoxin composed of human interleukin-13 (IL-13) fused to a mutated form of Pseudomonas aeruginosa exotoxin A (PE). This agent targets and kills tumor cells that express IL-13 receptors by inhibiting protein synthesis. Most malignant glioma cell lines and explants overexpress IL-13R $\alpha 2$ (2,6,7). Because IL-13PE is a powerful cytotoxic agent to IL-13R $\alpha 2$-expressing tumors, its activity was tested in preclinical and clinical studies of GBM (2). In several phase 1 and 2 clinical trials, 120 GBM patients were treated with IL-13PE by CED $(6,7)$. Infusion catheters were placed surgically into the tumor or surrounding brain parenchyma. On the basis of these results, a phase 3 clinical trial with intraparenchymal IL-13PE administration (PRECISE [Phase 3 Randomized Evaluation of CED of IL-13PE compare to Gliadel Wafer with Survival Endpoint in GBM]) was undertaken to compare carmustine-releasing Gliadel wafers (GW), an approved drug for newly diagnosed high-grade malignant glioma or recurrent GBM, as an adjunct to surgery in adult patients with GBM at first recurrence (6). GW has shown a small but significant improvement in survival (3). IL-13PE was well tolerated, but it did not show superiority in overall survival over GW. However, retrospective data analysis showed longer time to progression with IL-13PE than GW. It was hypothesized that the efficacy of IL-13PE by CED may have been reduced because of poor intracranial distribution or suboptimal catheter placement or catheter numbers $(8)$. 
In preclinical animal studies, IL-13PE distribution in the brain stem of nonhuman primates was tracked by coinfusion of gadolinium-bound albumin, followed by MR imaging and autoradiography of ${ }^{125}$ I-IL-13PE (9). This study did not evaluate binding and cytotoxic activity of the radiolabeled IL-13PE. For biodistribution and tracking of ${ }^{125} \mathrm{I}-\mathrm{IL}-13 \mathrm{PE}$, retention of biologic activity after radioiodination is important. Herein, we developed a biologically active and highly specific radioiodinated IL-13PE for administration into the brain parenchyma and intratumorally in an orthotopic glioma model. We observed better ${ }^{125}$ I-IL-13PE distribution by CED than bolus administration as assessed by micro-SPECT/CT.

\section{MATERIALS AND METHODS}

\section{Radioiodination of IL-13PE}

IL-13PE was radioiodinated by IODO-GEN (Thermo Scientific) and $\mathrm{Na}^{125}$ I (PerkinElmer) as described previously (10). The specific activity of the radiolabeled IL-13PE was $592.5 \pm 70.4 \mathrm{kBq} / \mu \mathrm{g}$.

\section{Characterization of ${ }^{125}$ I-IL-13PE}

For cytotoxicity assay, U251 and T98G human glioma cells $\left(5 \times 10^{5}\right)$ were plated in 100-mm Petri dishes with complete medium and cultured with ${ }^{125} \mathrm{I}-\mathrm{IL}-13 \mathrm{PE}(0-1,000 \mathrm{ng} / \mathrm{mL})$. To confirm specificity of the biologic activity, unlabeled IL-13 (100 ng/mL) was added before the addition of ${ }^{125} \mathrm{I}-\mathrm{IL}-13 \mathrm{PE}$. Viable cells were counted at the end of a 4-d culture by trypan blue exclusion technique.

For the receptor binding assay, equilibrium binding studies were performed as described previously (10). Cell-bound ${ }^{125} \mathrm{I}-\mathrm{IL}-13 \mathrm{PE}$ was separated by centrifugation through a cushion of phthalate oil and counted with a $\gamma$ counter.

To evaluate serum stability, ${ }^{125} \mathrm{I}-\mathrm{IL}-13 \mathrm{PE}(8.23 \mathrm{kBq} / 13.9 \mathrm{ng})$ in 25 $\mu \mathrm{L}$ of normal mouse serum were incubated in polypropylene tubes at $37^{\circ} \mathrm{C}$ for $0.5-24 \mathrm{~h}$. Bound or cleaved ${ }^{125} \mathrm{I}-\mathrm{IL}-13 \mathrm{PE}$ was separated after precipitating with $10 \%$ trichloroacetic acid, and radioactivity was measured with a $\gamma$ counter.

\section{Intracranial Orthotopic GBM Tumor Model}

Intracranial GBM tumors were developed in athymic nude mice using U251 glioma cells as described previously $(11,12)$. All in vivo procedures were approved by the National Cancer Institute/National Institutes of Health Animal Care and Use Committee (ACUC) and the Center for Biologics Evaluation and Research Institutional ACUC.

\section{Bolus and CED Administration of ${ }^{125}$ I-IL-13PE}

Twenty tumor-bearing mice received either bolus $(n=10)$ or CED $(n=10)$, and 5 normal mice underwent CED infusion. The bolus dose of ${ }^{125} \mathrm{I}$-IL-13PE was $925 \mathrm{kBq}$ in $5 \mu \mathrm{L}$ of phosphate-buffered saline containing $0.2 \%$ human serum albumin (HSA) and was injected through a preexisting burr hole at a depth of $2.5 \mathrm{~mm}$ below the surface of the skull via a Hamilton syringe. To avoid backflow and leakage, we infused the drug slowly over $10 \mathrm{~min} .{ }^{125} \mathrm{I}$-IL-13PE $(3,700 \mathrm{kBq}$ in 100 $\mu \mathrm{L}$ of phosphate-buffered saline/ $0.2 \%$ HSA) was infused at a rate of $1.4 \mu \mathrm{L} / \mathrm{h}$ over $3 \mathrm{~d}$ by a sterile osmotic pump (Durect Corp.) for CED $(11,12)$.

\section{SPECT/CT Imaging}

The mice were imaged at days 1,4 , and 7 after drug infusion by a micro-SPECT/CT device (Bioscan) using tomographic data acquisition in 10-20 s/projections for 30 projections. CT was acquired in 512 projections to allow anatomic coregistration. The energy peak for the camera was set to $28 \mathrm{keV}$, and the energy window was set to peak energy $\pm 18 \%$ (23-33 keV). Data reconstruction and analysis were performed with the ordered-subsets expectation maximization algorithm and InVivoScope1.42 software (Bioscan).

The volume of interest under the skull was manually drawn to determine the volume of distribution ( $\mathrm{Vd}$ ) using an automatic threshold-based algorithm for a threshold of $20 \%$ of the maximum counts within the whole-brain volume of interest. The threshold of $20 \%$ was found to be optimal by standard curves that were drawn by measuring $\mathrm{Vds}$ of a variety of known radioactivity with different thresholds (data not shown).

\section{Ex Vivo Biodistribution Study}

Eight tumor-bearing mice (bolus $n=4$ and $\operatorname{CED} n=4$ ) were euthanized at $1 \mathrm{~d}$ after completing the drug infusion, and the remaining 6 mice in each group were euthanized on day 7 after drug infusion. One of 5 normal mice was euthanized on day 1 , and the remaining 4 normal mice were euthanized on day 7 after infusing the drug. The vital organs and osmotic pumps were collected for radioactivity measurement and percentage injected dose (\%ID). The \%ID of the total blood volume was calculated by multiplying \% ID in a collected blood sample with the total blood volume using the equation: body weight $(\mathrm{g}) \times 79(\mu \mathrm{L} / \mathrm{g})(13)$.

\section{Autoradiography}

Four of 10 brain tumors from the bolus and CED groups were used for autoradiography. Two of 5 normal mice brains served as controls. The brains were embedded in sodium carboxymethyl cellulose matrix, frozen, and cut coronally at $320-\mu \mathrm{m}$ intervals rostral and caudal to the point of injection of the drug. Three serial sections from each coronal slice were fixed with acetone and sectioned on a cryostat (Leica Microsystems Inc.) at a thickness of $10 \mu \mathrm{m}$ each for hematoxylin and eosin analysis, total radioactivity, and autoradiography. Autoradiographic analysis was performed for $\mathrm{Vd}$ determination by exposing the sections to a storage phosphor plate (GE Healthcare Bio-Sciences). To define the boundaries of infusion, a threshold equal to approximately $15 \%$ of the maximum tissue equivalent was used (14).

\section{Statistical Analysis}

The data are presented as mean \pm SD. Statistical analysis was performed using the Student $t$ test for comparison between 2 groups and ANOVA among more than 2. The level of statistical significance was set at a $P$ value of 0.05 or less.

\section{RESULTS}

\section{Radioiodination and Characterization of ${ }^{125} \mathrm{I}-\mathrm{IL}-13 \mathrm{PE}$}

The cytotoxic activity of ${ }^{125} \mathrm{I}-\mathrm{IL}-13 \mathrm{PE}$ and IL-13PE was determined as shown in Figures 1A and 1B. We observed that radioiodination did not alter the cytotoxic activity of the molecule in receptorpositive or -negative glioma cell lines. The half maximal inhibitory concentration values were 0.8 and $0.9 \mathrm{ng} / \mathrm{mL}$ in U251 glioma cells for ${ }^{125} \mathrm{I}-\mathrm{IL}-13 \mathrm{PE}$ and IL-13PE, respectively. Further, the cytotoxic activity was completely neutralized by excess IL-13, indicating that ${ }^{125} \mathrm{I}-\mathrm{IL}-13 \mathrm{PE}$ cytotoxicity was highly specific. In binding assays, IL-13 also partially competed with ${ }^{125} \mathrm{I}-\mathrm{IL}-13 \mathrm{PE}$ binding (Figs. 1C and 1D).

Next, we examined the stability of ${ }^{125} \mathrm{I}-\mathrm{IL}-13 \mathrm{PE}$ in mouse serum to determine whether serum proteins or enzymes could degrade radiolabeled IL-13PE and cleave ${ }^{125} \mathrm{I}$ from the immunotoxin. As shown in Figure 1E, the trichloroacetic acid precipitation retained more than $75 \%$ of radioactivity up to 24 -h incubation at $37^{\circ} \mathrm{C}$, indicating that radioiodinated IL-13PE is highly stable in mouse serum.

\section{SPECT/CT Imaging}

The image acquisitions by micro-SPECT/CT on days 1,4 , and 7 were performed after infusion of ${ }^{125} \mathrm{I}$-IL-13PE. We observed a focal accumulation of ${ }^{125} \mathrm{I}-\mathrm{IL}-13 \mathrm{PE}$ in the brain tumor on day 1 after bolus injection. Uptake decreased on day 4 and further declined on day 7 (Fig. 2A). In contrast, CED images showed significantly higher accumulation of ${ }^{125} \mathrm{I}-\mathrm{IL}-13 \mathrm{PE}$ in the brain tumor on day 1 , which slowly cleared by day 7 , and this uptake was higher 


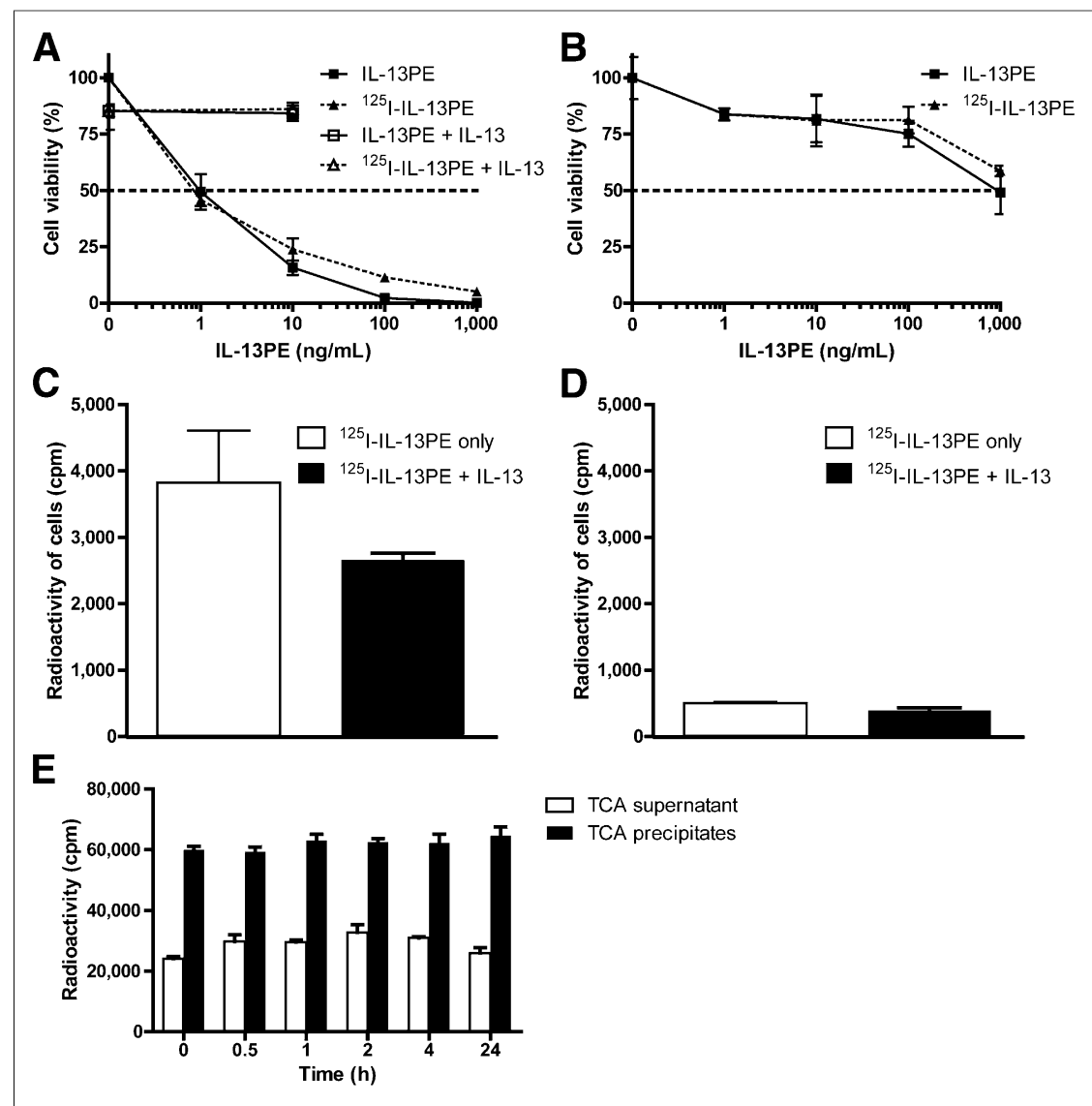

FIGURE 1. Radiolabeling and characterization of ${ }^{125}$ I-IL-13PE. Cytotoxicity of 125 I-IL-13PE to glioma cell lines U251 (A) or T98G (B) was assessed by incubating cells with different concentrations of ${ }^{125} \mathrm{I}-\mathrm{IL}-13 \mathrm{PE}$ for $4 \mathrm{~d}$. IL-13 $(100 \mathrm{ng} / \mathrm{mL})$ was used for blocking cytotoxicity of IL-13PE. Viable cells were counted by trypan blue exclusion. Binding of ${ }^{125} \mathrm{I}-\mathrm{IL}-13 \mathrm{PE}$ to U251 (C) or T98G (D) cells was assessed by incubating cells with ${ }^{125}$ I-IL-13PE $(100 \mathrm{pM})$ at $4^{\circ} \mathrm{C}$ for $16 \mathrm{~h}$ with or without 100 -fold excess of unlabeled IL-13. Cell-bound ${ }^{125}$ I-IL-13PE was separated by centrifugation through cushion of phthalate oil and counted by y counter. Stability of ${ }^{125}$ I-IL-13PE in mouse serum $(E)$ was determined by incubating with mouse serum at $37^{\circ} \mathrm{C}$ for $0.5-24 \mathrm{~h} .{ }^{125} \mathrm{I}-\mathrm{IL}-$ 13PE bound and free ${ }^{125}$ I were separated by trichloroacetic acid (TCA) precipitation. Radioactivity from protein precipitates and supernatant was measured by $y$ counter.

than with bolus administration (Figs. 2A and 2B). A similar pattern was observed for SPECT/CT images obtained after CED in normal brains, compared with brains with tumors (Figs. 2B and 2C). Similar patterns were also observed for maximum-intensity projections on day 1 after CED administration of IL-13PE with less radioactivity in the catheter and the least in the pump. Mild uptake was seen in the thyroid and bladder (Fig. 3).

\section{Biodistribution of ${ }^{125}$ I-IL-13PE After Bolus Injection or CED into Mouse Brain}

We harvested vital organs from mice undergoing bolus injection or CED and determined \%ID for each organ. All mice retained the highest radioactivity in the brain on day 1 and less than $1 \%$ radioactivity in other organs (Table 1). The values declined on day 7 in all groups of mice. Both CED-treated tumor-bearing or normal brains maintained significantly higher radioactivity on days 1 and 7 than brains treated by the bolus route $(P<0.001)$. No significant difference was observed in radioactivity between CED administered to tumorbearing brains or to normal brains during the course of the study.

The radioactivity in all vital organs and blood except the thyroid after CED decreased on the day 7, compared with day 1 . A similar pattern was observed when mice were injected by a bolus injection. Interestingly, the radioactivity was significantly higher on day 1 in the kidneys of mice treated by bolus route, compared with CED counterparts $(P<0.01)$. Although \% ID values of liver after bolus injection were higher than CED, the values were not statistically significant on day 1 but significantly higher on day 7 $(P<0.01)$. But the thyroids of all mice continued showing a nonsignificant increase in radioactivity for $7 \mathrm{~d}$. We did not see any significant difference in \%ID values in thyroid between bolus and CED-administered immunotoxin in tumor-bearing mice or normal mice, although statistics could not be determined because of a limited number of animals.

CED in tumor-bearing and normal brains showed retention of high radioactivity in the 3-d osmotic pump, which may occur because of the infusion rate difference caused by the osmotic pressure difference in the tissue environment.

\section{I-IL-13PE Distribution by Autoradiography in Mouse Brain}

To analyze SPECT/CT results for drug biodistribution in tumor-bearing and normal mouse brain, we performed autoradiography of brain sections after ${ }^{125} \mathrm{I}-\mathrm{IL}-13 \mathrm{PE}$ infusion (Fig. 4). We observed weak levels of ${ }^{125} \mathrm{I}-\mathrm{IL}-13 \mathrm{PE}$ in the tumor and around the needle track after bolus injection. In contrast, tumor sections from the CED group demonstrated higher concentrations of ${ }^{125} \mathrm{I}-\mathrm{IL}-13 \mathrm{PE}$ distributed throughout the tumor bed, which traveled along the external capsule without crossing the midline. Similarly, the autoradiography of normal brain after CED showed a higher accumulation and larger distribution of ${ }^{125} \mathrm{I}-\mathrm{IL}-13 \mathrm{PE}$.

Hematoxylin and eosin analysis of brains of glioma-bearing mice showed noticeable tumor-related neuropathologic changes, which were not specifically related cannula insertion. Normal brains did not show such histopathologic changes (Fig. 4).

\section{Tracking Accuracy of SPECT/CT for ${ }^{125}$ I-IL-13PE Distribution After Bolus or CED Injection in U251 Glioma-Bearing Mice}

We established the tracking accuracy of SPECT/CT for intracranial infusion by aligning the autoradiography with anatomic images. Autoradiography and SPECT/CT demonstrated similar anatomic epicenters of infusion (Fig. 4), and the Vd values were lower on SPECT/CT than autoradiography for actual distribution of ${ }^{125}$ I-IL13PE (Table 2). The Vd on day 7 after bolus injection was not measured by SPECT/CT because ${ }^{125} \mathrm{I}-\mathrm{IL}-13 \mathrm{PE}$ accumulation was undetectable. We observed the mean underestimation of actual infusion delivery by SPECT/CT (Vd values) to be $50.3 \% \pm 9.07 \%$.

\section{Vd of ${ }^{125}$ I-IL-13PE}

The Vd values by SPECT/CT were found to be significantly higher in tumors after CED of ${ }^{125} \mathrm{I}-\mathrm{IL}-13 \mathrm{PE}$ than bolus treatment on day 


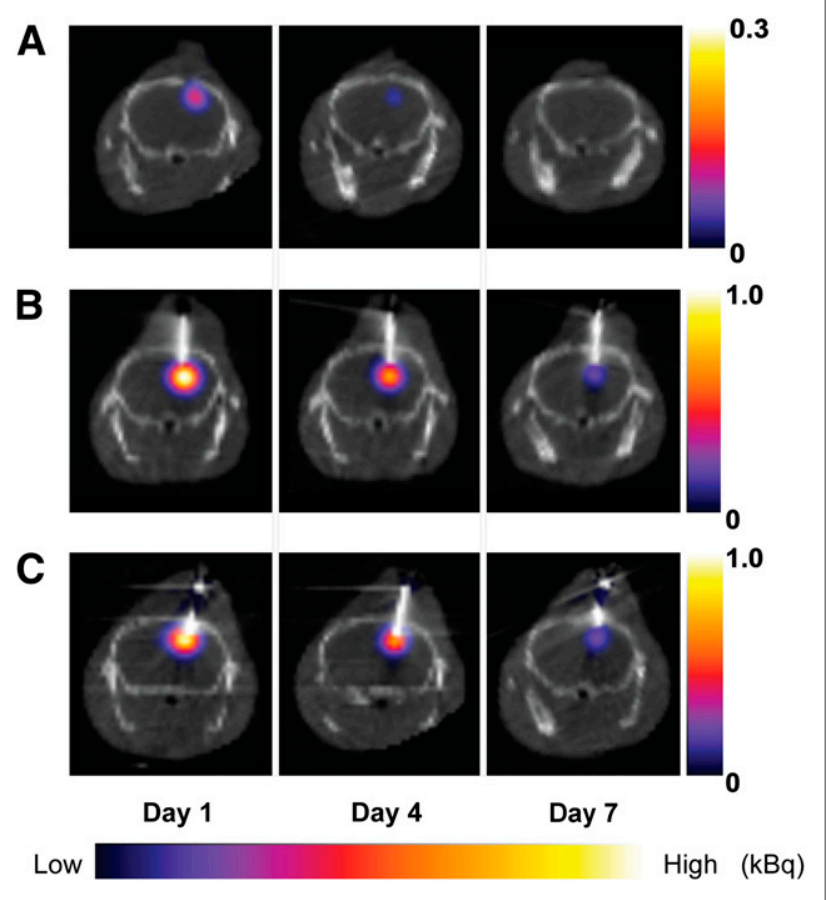

FIGURE 2. Coronal SPECT/CT images from mouse brain at days 1, 4, and 7 after ${ }^{125} \mathrm{I}-\mathrm{IL}-13 \mathrm{PE}$ drug infusion. Images after bolus injection showed focal accumulation of ${ }^{125} \mathrm{I}-\mathrm{IL}-13 \mathrm{PE}$ in brain tumor on day 1. Uptake decreased on day 4 and declined more on day 7 (A). Images after CED in both tumor-bearing and normal mouse showed accumulation of $125 \mathrm{I}$-IL13PE on day 1. Drug was found to be cleared by day 7 ( $B$ and $C$ ).

$1(P<0.001)$ (Table 3$)$. The Vd was $12.0 \pm 3.43 \mathrm{~mm}^{3}$ by CED in tumors and $10.9 \pm 4.97 \mathrm{~mm}^{3}$ in normal brains on day 7 by SPECT/CT, whereas the Vd determination was not done in mice treated by bolus injection on day 7 because of undetectable levels of ${ }^{125} \mathrm{I}$-IL-13PE. The Vds as determined by autoradiography in both CED groups (tumor and normal brain) were significantly higher than the bolus group on day $7(P<0.001)$ (Table 3$)$. The $\mathrm{Vd}$ of tumor-bearing brain after CED showed values similar to normal brain after CED on day 7.

\section{DISCUSSION}

We demonstrate that IL-13PE can be radioiodinated with $\mathrm{Na}^{125}$ I without affecting its biologic activity. IL-13R $\alpha 2$-positive but not -negative glioma cells were sensitive to ${ }^{125} \mathrm{I}-\mathrm{IL}-13 \mathrm{PE}$ and killed by subnanomolar concentrations of the immunotoxin. The cytotoxic activity of ${ }^{125} \mathrm{I}-\mathrm{IL}-13 \mathrm{PE}$ was identical to unlabeled IL-13PE, indicating that radiolabeling did not change its functional activity. Our results further demonstrate that an excess of IL-13 could completely neutralize the cytotoxicity of ${ }^{125} \mathrm{I}-\mathrm{IL}-13 \mathrm{PE}$ and IL-13PE, but it only partially inhibited ${ }^{125} \mathrm{I}-\mathrm{IL}-13 \mathrm{PE}-$ specific binding to IL-13R $\alpha 2$-expressing tumor cells. This partial inhibition may be explained in part by stearic hindrance caused by fusion of PE to IL-13 or radioiodination of IL13PE, which did not allow high-affinity binding of ${ }^{125} \mathrm{I}-\mathrm{IL}-13 \mathrm{PE}$ to U251 cells, and thus IL-13 could not completely displace ${ }^{125} \mathrm{I}-\mathrm{IL}-$ 13PE. In contrast, the cytotoxicity is not completely dependent on the binding affinity of IL-13PE to IL-13 receptor on target cells. It has been demonstrated that 1 molecule of PE when internalized is sufficient to kill the cells. Because of this high sensitivity of target cells to only a few molecules of IL-13PE, excess IL-13 is able to completely inhibit its cytotoxicity.
Our studies also demonstrate that ${ }^{125} \mathrm{I}-\mathrm{IL}-13 \mathrm{PE}$ maintains its stability in mouse serum for up to $24 \mathrm{~h}$ of incubation examined, indicating that IL-13PE will remain intact during biodistribution and therapy studies. The stability of IL-13-PE in serum is also supported by studies in which ${ }^{125}$ I-IL-13-PE was highly cytotoxic to IL-13 receptorpositive tumor cells, which were incubated with IL-13PE in the presence of bovine serum for $5 \mathrm{~d}$ (Fig. 1A). Thus, ${ }^{125} \mathrm{I}$-IL-13PE may be a useful agent for intracranial biodistribution studies in vivo.

SPECT/CT studies revealed that ${ }^{125} \mathrm{I}-\mathrm{IL}-13 \mathrm{PE}$ is retained in gliomas for a prolonged period when administered by CED, compared with bolus injection. Autoradiography results corroborated the CED data, which showed 5.9 and 16.9 times higher Vd on days 1 and 7, respectively, than bolus injection. The mice were treated with a 4-foldhigher injection dose (ID) by CED than bolus injection (3,700 vs. $925 \mathrm{kBq}$ ). When ID values were normalized, CED showed 1.5 times higher Vd on day 1 and 4.2 times higher Vd on day $7(P<0.05$ and $0.01)$, compared with bolus injection. In vivo results also corroborated with previous clinical studies, which showed that CED is far superior in drug distribution within the tumor and brain parenchyma, compared with bolus administration. In addition, it has been demonstrated that CED infusions are well tolerated in glioma models $(11,14)$.

In our previous study, we assessed the distribution kinetics of IL-13PE in U251 tumor-bearing mice indirectly after bolus injection and CED by performing immunohistochemical and Western blot analyses with anti-IL-13 and anti-PE antibodies (11). However, in the present study, we directly report biodistribution of ${ }^{125} \mathrm{I}-$ IL-13PE and demonstrate that higher Vds of IL-13PE can be achieved in mouse brains by CED rather than by the bolus route, which also corroborates with immunohistochemistry studies. We also show that IL-13PE is retained for a longer duration with a higher volume of distribution in intracranial tumors. This may be due to differences in ID, injection parameters, and sensitivity of the detection methods. Because in vitro studies showed that ${ }^{125} \mathrm{I}-\mathrm{IL}-$ 13PE maintained its binding/functional activity and stability in mouse serum, it was less likely that ${ }^{125}$ I may have been cleaved during or after drug infusion, resulting in nonspecific assessment of the drug distribution. The superiority of CED was confirmed by $\gamma$-scintillation

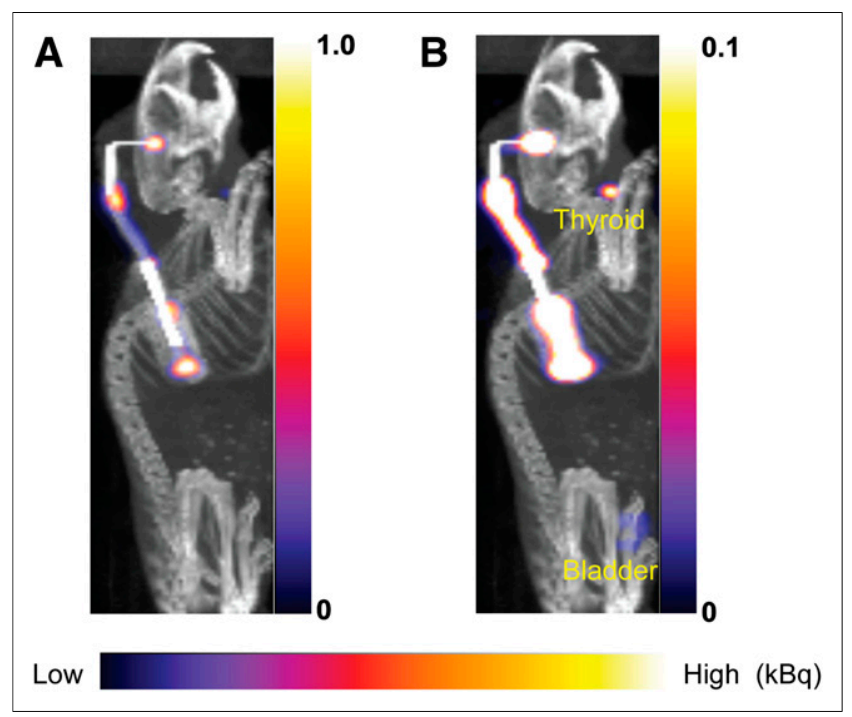

FIGURE 3. Maximum-intensity-projection SPECT/CT images from tumorbearing mouse on day 1 after CED and shown on color scale. Images showed high uptake in brain, catheter tube, and osmotic pump (A). Mild uptake was seen in thyroid and bladder (B). 
TABLE 1

Radiolocalization of ${ }^{125}$ I-IL-13PE After Bolus Injection or CED

\begin{tabular}{|c|c|c|c|c|c|c|}
\hline \multirow[b]{2}{*}{ Organ } & \multicolumn{2}{|c|}{ Bolus injection in tumor } & \multicolumn{2}{|c|}{ CED in tumor } & \multicolumn{2}{|c|}{ CED in normal brain } \\
\hline & day $1(n=3)$ & day $7(n=3)$ & day $1(n=3)$ & day $7(n=3)$ & day $1(n=1)$ & day $7(n=2)$ \\
\hline Brain & $2.787 \pm 0.192^{*}$ & $0.549 \pm 0.276^{\dagger}$ & $4.155 \pm 0.129^{*}$ & $1.057 \pm 0.102^{\dagger}$ & 3.985 & $1.016 \pm 0.095^{\dagger}$ \\
\hline Thyroid & $0.328 \pm 0.169$ & $0.780 \pm 1.164$ & $0.522 \pm 0.277$ & $1.021 \pm 0.252$ & 0.917 & $0.874 \pm 0.392$ \\
\hline Blood & $0.818 \pm 0.518^{\ddagger}$ & $0.021 \pm 0.010^{\ddagger}$ & $0.260 \pm 0.054$ & $0.025 \pm 0.007$ & 0.615 & $0.037 \pm 0.020$ \\
\hline Kidney & $0.252 \pm 0.088^{\S \|}$ & $0.048 \pm 0.011^{\S}$ & $0.117 \pm 0.017 \|$ & $0.065 \pm 0.016$ & 0.157 & $0.091 \pm 0.030$ \\
\hline Liver & $0.808 \pm 0.683$ & $0.160 \pm 0.032^{\uparrow}$ & $0.131 \pm 0.054$ & $0.026 \pm 0.0079$ & 0.198 & $0.064 \pm 0.0139$ \\
\hline Spleen & $0.048 \pm 0.036$ & $0.012 \pm 0.004$ & $0.010 \pm 0.003$ & $0.002 \pm 0.001$ & 0.014 & $0.006 \pm 0.003$ \\
\hline Heart & $0.011 \pm 0.001$ & $0.002 \pm 0.002$ & $0.009 \pm 0.003$ & $0.001 \pm 0.001$ & 0.017 & $0.001 \pm 0.001$ \\
\hline Pump & & & $14.03 \pm 4.644$ & $15.09 \pm 0.335$ & 14.74 & $12.22 \pm 0.550$ \\
\hline
\end{tabular}

counting of tissues. The uptake of ${ }^{125}$ I-IL-13PE in brain after CED was greater than bolus injection on day 1 and day 7 .

Rapid clearance of radioactivity of drug from the blood and kidney was observed after bolus injection, perhaps because of its excretion into urine. However, this clearance was delayed in the CED group because of its slow infusion over a period of $3 \mathrm{~d}$. Our results of radiolocalization of the drug after bolus and CED injections suggested that \%ID values of liver after bolus injection were slightly higher than CED, but the values were not statistically significant on day 1 . However, we observed \%ID values of liver after bolus injection that were significantly higher on day 7 than CED values, even though a higher amount of radioactivity was administered by CED. This may be because CED ${ }^{125 I}$-IL13-PE was infused over a prolonged period of time. The second highest accumulation of radioactivity was found in the thyroid of all groups of mice. The uptake of radioactivity was also observed in the thyroid and the bladder with SPECT/CT images. Taken together, these data suggest that ${ }^{125} \mathrm{I}-\mathrm{IL}-13 \mathrm{PE}$ injected into the brain may have been cleared into cerebrospinal fluid, entered the vascular system through the dural venous sinuses via the arachnoid granulations, and was metabolized and excreted into the urinary system. During this process, free ${ }^{125} \mathrm{I}$ may have accumulated in the thyroid. However, total radioactivity in all organs including thyroid comprised less than $1.6 \%-2.2 \%$ of total injected dose on day 7 whereas in the thyroid it comprised only $0.8 \%-1.0 \%$. Consistent with very low radioactivity, we did not observe any visible toxicity to any vital organs including the thyroid. In a phase 3 clinical trial when IL-13PE was administered intracranially, we did not observe any thyroid toxicities, most likely due to lack of expression of IL-13R $\alpha 2$ in the thyroid (7).

Interestingly, ${ }^{125} \mathrm{I}-\mathrm{IL}-13 \mathrm{PE}$ was also distributed in the normal brain after CED, in a manner similar to tumor-bearing brain. Autoradiography data showed that ${ }^{125} \mathrm{I}-\mathrm{IL}-13 \mathrm{PE}$ was distributed at the injection site in the normal brain. Although the Vd in tumor was higher than normal brain on day 7 after injection, the values were not statistically significant. Also, there was no significant difference in \%ID between tumorbearing brain and normal brain on day 1 and day 7 , suggesting that CED can maintain high concentrations of ${ }^{125} \mathrm{I}-\mathrm{IL}-13 \mathrm{PE}$ in the normal brain. This may be because of slower infusion of ${ }^{125} \mathrm{I}-\mathrm{IL}-13 \mathrm{PE}$, resulting in delayed clearance from the brain. Similar observations were reported by various investigators studying drug distribution in the normal brain of rodents after CED using radiolabeled markers in which they used radiolabeled large molecules such as albumin and epidermal growth factor and reported high Vd over longer periods in the normal brain (14).

We found that SPECT/CT does not accurately track convection of ${ }^{125} \mathrm{I}-\mathrm{IL}-13 \mathrm{PE}$ in mouse brain, which may be due to its low spatial resolution. In contrast, autoradiography seemed to be accurate because of its higher spatial resolution (a few hundred microns vs. a few millimeters by SPECT/CT) (15). Nevertheless, SPECT/CT has several major advantages over other modalities (e.g., optical and MR imaging) such as its higher sensitivity at

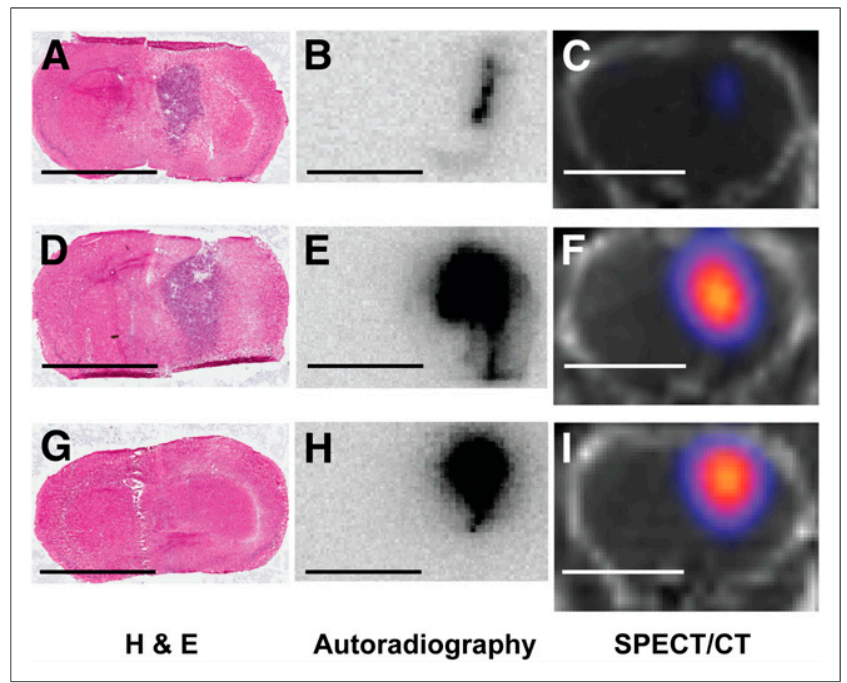

FIGURE 4. Hematoxylin and eosin-stained coronal sections, corresponding autoradiographs, and SPECT/CT images from center of injection site of U251 glioma-bearing mouse (A-F) and normal brain (G-I). Mice were euthanized on day 7 after either bolus (A-C) or CED (D-I) of 125I-IL-13PE injection, and frozen brains were sectioned coronally. U251 glioma tumors were seen in implanted site in cortex and spread into caudate $(A$ and $D)$. Autoradiography after bolus injection displayed weak levels of ${ }^{125} \mathrm{I}-\mathrm{IL}-13 \mathrm{PE}$ in tumor and around needle track (B), and after CED in tumor-bearing mouse showed that high concentrations of 125 IL-13PE distributed throughout tumor and traveled along external capsule (E). Autoradiography after CED in normal brain showed high concentrations of ${ }^{125} \mathrm{I}-\mathrm{IL}-13 \mathrm{PE}$ distributed at injection site $(\mathrm{H})$. Corresponding SPECT/CT images demonstrated similar anatomic epicenters of infusion $(C, F$, and I). Bar $=5 \mathrm{~mm}$. H \& E = hematoxylin and eosin. 
TABLE 2

Tracking Accuracy of SPECT/CT for ${ }^{125}$ I-IL-13PE Distribution After Bolus Injection or CED into Glioma-Bearing Mouse Brain

\begin{tabular}{lccccc}
\hline & & & & & SPECT \\
\cline { 4 - 6 } Animal no. & Injection method & Time after injection & Autoradiography, Vd $\left(\mathrm{mm}^{3}\right)$ & Vd $\left(\mathrm{mm}^{3}\right)$ & $\begin{array}{c}\text { Percentage difference with } \\
\text { autoradiography }\end{array}$ \\
\hline 1 & Bolus & Day 1 & 9.78 & 4.10 & -41.9 \\
\hline 2 & Bolus & Day 7 & 1.51 & Not available & Not available \\
\hline 3 & Bolus & Day 7 & 0.72 & Not available & Not available \\
\hline 5 & Bolus & Day 7 & 1.04 & Not available & Not available \\
\hline 7 & CED & Day 1 & 36.6 & 20.3 & -55.4 \\
\hline 8 & CED & Day 7 & 15.7 & 8.19 & -52.2 \\
\hline
\end{tabular}

the picomolar probe level and desired tissue penetration. Additionally, because of the considerable differences in volume between humans and mice $(\sim 1,500$ vs. $0.5 \mathrm{~g})$, SPECT/CT is powerful enough to assess the human brain and acquire better-quality images than autoradiographic images of mouse brains. Sampson et al. have shown distribution of ${ }^{123}$ I-HSA after CED in patients with recurrent malignant gliomas by SPECT to delineate the infusion and calculate $\mathrm{Vd}$ (16) and showed that SPECT could be useful in tracking the drug distribution in the patients. Additional options of radiolabeling of IL-13PE with other radioiodines such as ${ }^{123} \mathrm{I}$ (half-life, $13 \mathrm{~h}$ ) or ${ }^{124} \mathrm{I}$ (half-life, $4.18 \mathrm{~d}$ ) by similar techniques may allow meaningful SPECT or PET imaging, respectively, in future clinical studies.

The CT component of SPECT/CT provides anatomic information, and SPECT/CT enables a direct correlation of anatomic and functional information, resulting in better localization and definition of scintigraphic findings. It is likely that the diagnostic values of combined SPECT/CT examinations of the brain tumor remain limited. A combined approach of merging MR images and SPECT/CT images may provide meaningful information to diagnose recurrent GBM. Future studies can be a combinatorial strategy of PET/MR imaging and SPECT/MR imaging to enhance the resolution for precise diagnosis and prognosis.

We considered that the use of an osmotic pump may not be the ideal method to perform CED in a rodent model and cannot directly extrapolate preclinical results of mouse models to larger animals or humans because of the size of the brain. However, the main objective of our study was to demonstrate that IL-13PE can be radiolabeled with ${ }^{125} \mathrm{I}$ without loss of its biologic activity and that it can be administered by CED to yield a higher Vd than with the bolus route. Additionally, our results support previous conclusions that orthotopic murine models of human glioma are the best models that mimic the clinical condition of glioblastoma. Furthermore, SPECT analysis of radiolabeled drug has been widely used to evaluate drug distribution in these models (17).

Furthermore, limitations of using osmotic pumps for biodistribution of IL-13PE in orthotopic mouse models of human glioma can be overcome using external pumps with catheters inserted stereotactically to demonstrate the safety, efficacy, and biodistribution in GBM patients $(6,7)$.

Previously, ${ }^{123}$ I-albumin and gadolinium-albumin tracers have been used to assess drug distribution by CED when coadministered with IL-13PE $(9,16)$. But, this approach may not be comparable from the actual distribution of IL-13PE because of differences in their molecular weights (albumin, $67 \mathrm{kDa}$ vs. IL-13PE, $52 \mathrm{kDa}$ ) and binding kinetics. Because of higher binding specificity and cytotoxic activity of ${ }^{125} \mathrm{I}-\mathrm{IL}-13 \mathrm{PE}$, our data may more accurately reflect IL13PE biodistribution in vivo for drug delivery in patients with GBM. We also observed that intracranial IL-13PE administration

TABLE 3

Vd of ${ }^{125}$ I-IL-13PE After Bolus Injection or CED into Mouse Brain

\begin{tabular}{|c|c|c|c|c|c|c|c|c|}
\hline \multirow[b]{2}{*}{ Group } & \multirow[b]{2}{*}{ Day } & \multicolumn{3}{|c|}{ Injection parameter } & \multicolumn{2}{|c|}{ SPECT } & \multicolumn{2}{|c|}{ Autoradiography } \\
\hline & & Time & $\begin{array}{c}\text { Volume of } \\
\text { injection }(\mu \mathrm{L})^{\star}\end{array}$ & ID (kBq) & $\mathrm{Vd}\left(\mathrm{mm}^{3}\right)$ & $\begin{array}{l}\text { No. of } \\
\text { mice }\end{array}$ & $\mathrm{Vd}\left(\mathrm{mm}^{3}\right)$ & $\begin{array}{l}\text { No. of } \\
\text { mice }\end{array}$ \\
\hline \multirow[t]{2}{*}{ Bolus injection in tumor } & 1 & $10 \min$ & 5 & 925 & $3.11 \pm 0.70^{\star}$ & 4 & 9.78 & 1 \\
\hline & 7 & $10 \min$ & 5 & 925 & Not available & 6 & $1.09 \pm 0.40^{\dagger}$ & 3 \\
\hline \multirow[t]{2}{*}{ CED in tumor } & 1 & $3 d$ & 100 & 3,700 & $18.3 \pm 1.37^{\star}$ & 4 & 36.6 & 1 \\
\hline & 7 & $3 d$ & 100 & 3,700 & $12.0 \pm 3.43$ & 6 & $18.4 \pm 2.41^{\dagger}$ & 3 \\
\hline \multirow[t]{2}{*}{ CED in normal brain } & 1 & $3 d$ & 100 & 3,700 & 11.7 & 1 & Not available & 0 \\
\hline & 7 & $3 d$ & 100 & 3,700 & $10.9 \pm 4.97$ & 4 & $14.3 \pm 5.42^{\dagger}$ & 2 \\
\hline
\end{tabular}

${ }^{*}$, tSignificant difference between groups $(P<0.001)$.

${ }^{*}$ Normalized $\mathrm{Vd}$ of CED is $4.58 \pm 0.34$ in tumor on day $1(P<0.05)$.

${ }^{\dagger}$ Normalized Vds of CED are $4.60 \pm 0.60$ in tumor and $3.56 \pm 1.36$ in normal brain on day $7(P<0.01)$. 
in GBM patients was well tolerated without systematic or localized toxicity $(6,7)$. Moreover, IL-13PE can be labeled with ${ }^{131} \mathrm{I}$, which can emit $\beta$ particles for targeted radiotherapy. Previously, ${ }^{131}$ I-labeled monoclonal antibodies have been administered directly into surgically created tumor resection cavities to potentially sterilize the margins of the tumor (18). Similarly, ${ }^{131} \mathrm{I}$-IL-13PE can be conjugated and delivered directly into the tumor bed following a similar protocol used in the phase 3 PRECISE trial to enhance the antitumor effect of IL-13PE with $\beta$ irradiation.

\section{CONCLUSION}

IL-13PE can be successfully radiolabeled with ${ }^{125}$ I without loss of its biologic activity and can be administered by CED for better biodistribution. In future trials, delivery of IL-13PE or other drugs via intracranial CED may benefit the GBM patients and provide additional data of biodistribution for better drug delivery in patients.

\section{DISCLOSURE}

The costs of publication of this article were defrayed in part by the payment of page charges. Therefore, and solely to indicate this fact, this article is hereby marked "advertisement" in accordance with 18 USC section 1734 . This project was supported by an appointment of Akiko Suzuki to the Research Participation Program at the Center for Biologics Evaluation and Research administered by the Oak Ridge Institute for Science and Education. No other potential conflict of interest relevant to this article was reported.

\section{ACKNOWLEDGMENTS}

We thank Stephen Adler and Mark Williams for their help in microSPECT/CT and autoradiography, Miriam Anver for histologic analysis, and Mary Custer and Christine Sadr of the NCI AUCU committee for their help with animals.

\section{REFERENCES}

1. Omuro A, DeAngelis LM. Glioblastoma and other malignant gliomas: a clinical review. JAMA. 2013;310:1842-1850.
2. Shimamura T, Husain SR, Puri RK. The IL-4 and IL-13 pseudomonas exotoxins: new hope for brain tumor therapy. Neurosurg Focus. 2006;20:E11.

3. Brem H, Piantadosi S, Burger PC, et al. Placebo-controlled trial of safety and efficacy of intraoperative controlled delivery by biodegradable polymers of chemotherapy for recurrent gliomas. The Polymer-brain Tumor Treatment Group. Lancet. 1995;345:1008-1012.

4. Lidar Z, Mardor Y, Jonas T, et al. Convection-enhanced delivery of paclitaxel for the treatment of recurrent malignant glioma: a phase I/II clinical study. J Neurosurg. 2004;100:472-479.

5. Rainov NG, Heidecke V. Long term survival in a patient with recurrent malignant glioma treated with intratumoral infusion of an IL4-targeted toxin (NBI3001). J Neurooncol. 2004;66:197-201.

6. Kunwar S, Chang S, Westphal M, et al. Phase III randomized trial of CED of IL13-PE38QQR vs Gliadel wafers for recurrent glioblastoma. Neuro Oncol. 2010;12:871-881

7. Kunwar S, Prados MD, Chang SM, et al. Direct intracerebral delivery of cintredekin besudotox (IL13-PE38QQR) in recurrent malignant glioma: a report by the Cintredekin Besudotox Intraparenchymal Study Group. J Clin Oncol. 2007; 25:837-844.

8. Mueller S, Polley MY, Lee B, et al. Effect of imaging and catheter characteristics on clinical outcome for patients in the PRECISE study. J Neurooncol. 2011;101: 267-277.

9. Murad GJ, Walbridge S, Morrison PF, et al. Real-time, image-guided, convectionenhanced delivery of interleukin 13 bound to pseudomonas exotoxin. Clin Cancer Res. 2006;12:3145-3151.

10. Husain SR, Joshi BH, Puri RK. Interleukin-13 receptor as a unique target for anti-glioblastoma therapy. Int J Cancer. 2001;92:168-175.

11. Kawakami K, Kawakami M, Kioi M, Husain SR, Puri RK. Distribution kinetics of targeted cytotoxin in glioma by bolus or convection-enhanced delivery in a murine model. J Neurosurg. 2004;101:1004-1011.

12. Kawakami K, Kioi M, Liu Q, Kawakami M, Puri RK. Evidence that IL-13R alpha2 chain in human glioma cells is responsible for the antitumor activity mediated by receptor-directed cytotoxin therapy. J Immunother. 2005;28:193-202.

13. Harkness JEWJ. Biology and husbandry. In: Harkness and Wagner's Biology and Medicine of Rabbits and Rodents. 5th ed. Ames, IA: Wiley-Blackwell; 2010.

14. Yang W, Barth RF, Adams DM, et al. Convection-enhanced delivery of boronated epidermal growth factor for molecular targeting of EGF receptor-positive gliomas. Cancer Res. 2002;62:6552-6558.

15. Schmidt KC, Smith CB. Resolution, sensitivity and precision with autoradiography and small animal positron emission tomography: implications for functional brain imaging in animal research. Nucl Med Biol. 2005;32:719-725.

16. Sampson JH, Raghavan R, Brady ML, et al. Clinical utility of a patient-specific algorithm for simulating intracerebral drug infusions. Neuro-oncol. 2007;9:343-353.

17. de Jong M, Maina T. Of mice and humans: are they the same? Implications in cancer translational research. J Nucl Med. 2010;51:501-504.

18. Zalutsky MR. Current status of therapy of solid tumors: brain tumor therapy. J Nucl Med. 2005;46(suppl 1):151S-156S. 